DIAS-STP-94-21

June 10th 1994

\title{
Symplectic Geometry And Hamiltonian Flow Of The Renormalisation Group Equation
}

\author{
Brian P. Dolan \\ Department of Mathematical Physics, St. Patrick's College \\ Maynooth, Ireland \\ and \\ Dublin Institute for Advanced Studies \\ 10, Burlington Rd., Dublin, Ireland \\ e-mail:bdolan@maths.may.ie
}

\begin{abstract}
It is argued that renormalisation group flow can be interpreted as being a Hamiltonian vector flow on a phase space which consists of the couplings of the theory and their conjugate "momenta", which are the vacuum expectation values of the corresponding composite operators. The Hamiltonian is linear in the conjugate variables and can be identified with the vacuum expectation value of the trace of the energy-momentum operator. For theories with massive couplings the identity operator plays a central role and its associated coupling gives rise to a potential in the flow equations. The evolution of any quantity, such as $N$-point Green functions, under renormalisation group flow can be obtained from its Poisson bracket with the Hamiltonian. Ward identities can be represented as constants of the motion which act as symmetry generators on the phase space via the Poisson bracket structure.
\end{abstract}




\section{$\S 1$ Introduction}

The history of relativistic quantum field theory is plagued with divergences. The canonical cure for this is to replace the (infinite) bare co-ordinates $g_{0}^{a}$ by (finite) renormalised co-ordintates $g_{R}^{a}(\kappa)$ at some renormalisation point $\kappa$. This requires regulating the divergences and choosing a subtraction prescription by introducing counter terms. Even for theories with no divergences it is sometimes useful to introduce counter terms and define renormalised couplings - an example of this is the $\epsilon$-expansion around the Wilson-Fisher non-trivial fixed point in 3-dimensional massive $\varphi^{4}$ theory [1]

However knowledge of the renormalised couplings alone is not sufficient, one must also know what the counter terms are. This is (almost) equivalent to knowing the $\beta$-functions of the theory, since the counter terms are specified by the difference $\Delta g^{a}(\kappa)=g_{0}^{a}-g_{R}^{a}(\kappa)$. Demanding that the bare couplings are independent of the renormalisation point gives

$$
\beta^{a}=\frac{d g_{R}^{a}}{d t}=-\frac{d\left(\Delta g^{a}\right)}{d t}
$$

where $t=\ln \kappa$.

The traditional approach has been first to choose the counter terms and then calculate the $\beta$-functions. However the counter terms contain a certain ambiguity, they can be modified by adding a finite function of the couplings to $\Delta g^{a}$. Thus, as is well known, the $\beta$-functions are not unique, they depend on the subtraction procedure. In practice though they are constrained by the requirement that a renormalisation prescription be chosen which leads to a perturbation theory that makes sense i.e. converges reasonably quickly, at least asymptotically. Of course, the $\beta$-functions are not completely arbitrary - they have zeros which cannot be removed by changing prescription. Viewing them as vector fields ("velocities") on the space of couplings, a zero of the vector field in one prescription remains a zero in any other prescription (of course individual components of the vector $\vec{\beta}$ may vanish in one prescription and not in another, but a zero of the vector field requires the entire vector to vanish). Thus, in one sense at least, a change in renormalisation prescription can be thought of as a co-ordinate transformation (diffeomorphism) on the space of couplings since this also leaves the zeros of a vector field unchanged (although it may, and in general would, change the numerical values of the co-ordinates of the point at which the zero occurs).

One can imagine turning the logic round and first choosing the $\beta$-functions and then using equation (1) to determine the counter terms (up to an arbitrary constant i.e. a renormalisation group invariant). Of course the choice must be judicious - it would be crazy to choose a positive $\beta$-function for massless QCD, the resulting theory would be completely unstable and probably would not even exist. In theories with more than one coupling, however, there is more freedom. If one were unlucky enough to choose a $\beta$ function which pointed away from a nearby attractive fixed point the RG evolution would presumably force it to turn round and point in towards the fixed point - behaviour which cannot happen if there is only one coupling. Choosing a $\beta$-function with the "wrong sign" in a theory with more than one coupling is not pathological, it is merely an indication of a "bad" choice of co-ordinates. Transforming to "good" co-ordinates (i.e. co-ordinates 
which have a sensible physical interpretation and are not just abstract parameters) would require performing a diffeomorphism which "unwinds" the RG trajectory so that it flows into the fixed point in a straight line. In actual fact, from a topological point of view, the notion of "pointing away" does not make sense unless a metric is defined on the space of couplings. To have a concept of "pointing away" requires having a definition of angles, and this needs a metric. Without a metric any direction is much the same as any other, it is difficult to navigate in a space without a metric!

Another quantity which should not change under a co-ordinate transformation is the signature of the matrix of anomalous dimensions, $\partial_{a} \beta^{b}$, at a fixed point. The number of positive and negative eigenvalues of this matrix determines in how many directions the RG flow is attracted to the fixed point and in how many it is repelled from it. For massless QCD this matrix is one dimensional and this is another way to see that the sign of the $\beta$-function cannot be changed by a co-ordinate transformation in a theory with only one coupling.

There is an analogy here with classical mechanics. Consider a theory with $n-1$ couplings, $g^{a}, a=1, \ldots, n-1$ (from now on the subscript $R$ on couplings will be dropped in this section - all couplings are renormalised unless otherewise indicated). Denote the space of couplings by $\mathcal{M}$. The $\beta$-functions $\beta^{a}(g)$ constitute a vector field on the $n-1$-dimensional differentiable manifold $\mathcal{M}$ (for the moment the topology and global properties of $\mathcal{M}$ will not be relevant and it may as well be taken to be $\mathbf{R}^{n-1}$ - consideration of the global topology will be restricted to a few comments in the final section). The $2 n-2$ dimensional space with co-ordinates $\left(g^{a}, \beta^{a}\right)$ (the tangent bundle $T(\mathcal{M})$ ) is thus analogous to the configuration space of co-ordinates and velocities in classical mechanics. Choose a point in $T(\mathcal{M})$ and the evolution of the system is determined by the dynamics. All the necessary information for computing the RG evolution is contained in the generating functional (or free energy in statistical mechanics) $W(g, t)=\int w(g, t) d^{D} x=-\ln Z$ where $w(g, t)$ is the free energy density. * Just as in classical mechanics one can imagine transforming to a phase space, with co-ordinates $\left(g^{a}, \phi_{a}\right)$ (the cotangent bundle $T^{*}(\mathcal{M})$ ) where $\phi_{a}$ are "momenta" dual to the "velocities" $\beta^{a}$. A natural choice for the $\phi_{a}$ is the vacuum expectation value of the (in general composite) operator associated with the coupling $g^{a}, \phi_{a}=\frac{\partial w(g, t)}{\partial g^{a}}[2]$. In order to streamline some of the formula it will be convenient to re-scale all the couplings by their canonical dimensions so that they are dimensionless. When this is done the variables $\phi_{a}$ are densities with canonical mass dimension $D$.

Following O'Connor and Stephens [2] one can ask if there might be some notion of a Hamiltonian function on phase space $H(g, \phi, t)$ which could govern the RG evolution of the couplings and the expectation values together so that the RG flow can be regarded as a Hamiltonian vector flow on phase space. The answer is yes and the construction turns out to be remarkably simple. A Hamiltonian will be presented in section three which is linear in the momenta, rather than quadratic as in non-relativistic particle mechanics. It is in fact minus the expectation value of the trace of the energy-momentum operator,

* For simplicity we shall work on flat $D$-dimensional Euclidean space, so that translational invariance ensures that $w(g, t)$ is independent of position, but the concepts presented here are more general than this. 
$H=-\langle T\rangle$. Despite the linearity of the Hamiltonian in the momenta, the dynamical evolution is not trivial and some examples will be examined to show this in detail.

The construction requires a careful consideration of the role of the identity operator in theories which involve massive couplings. This can be done by introducing a coupling associated with the identity operator, $\Lambda$ (a cosmological constant). Its conjugate momentum, $\phi_{\Lambda}$, is just the expectation value of the identity operator. The $\beta$-function associated with $\Lambda$ turns out to be linear in $\Lambda[3], \beta^{\Lambda}(g, \Lambda)=-D \Lambda+U(g)$ where $U(g)$ is an analytic function of the other couplings and is independent of $\Lambda$. The conventions adopted in this paper are such that the cosmological constant is scaled by its canonical dimensions and $\Lambda$ is dimensionless. This explains the term $-D \Lambda$ here which not otherwise be present. A consequence of this is that the $\phi_{\Lambda}=\kappa^{D}$ is also a density. For massless theories, $U(g)=0$. For the purposes of the introductory discussion presented here it will be assumed that the $\beta$-functions have no explicit $\kappa$ dependence and only depend on $\kappa$ implicitly through $g^{\hat{a}}(\kappa)$. A full generalisation to the situation where a subtraction procedure is chosen in which the $\beta$-functions have explicit $\kappa$ dependence is given later.

It will be shown in section three that the Hamiltonian defined by

$$
H(g, \phi)=\beta^{a}(g) \phi_{a}+\beta^{\Lambda}(g, \Lambda) \phi_{\Lambda}
$$

governs the renormalisation group flow of the couplings $g^{a}$ and the expectation values $\phi_{a}$. Of course, once the theory has been solved, all of the vacuum expectation values (VEV's) can be expressed as functions of $g^{a}$ and $t, \phi_{a}=\phi_{a}(g, t)$ and then the Hamiltonian can indeed then be written as a function of $g^{a}, \Lambda$ and $t$ alone (for example $\phi_{\Lambda}=\kappa^{D}$ ). However the philosophy here is that at the outset $g^{a}$ and $\phi_{a}$ are to be considered as independent variables and $H(g, \phi)$ depends on each separately.

The main result of this paper is that the RG evolution of $g^{a}$ and $\phi_{a}$ is given by "Hamilton's equations",

$$
\begin{aligned}
\frac{d g^{a}}{d t} & =\left.\frac{\partial H}{\partial \phi_{a}}\right|_{g} \\
\frac{d \phi_{a}}{d t} & =-\left.\frac{\partial H}{\partial g^{a}}\right|_{\phi}
\end{aligned}
$$

The first equation follows simply from the definition of $H(g, \phi)$ in $(2)$ while the second contains non-trivial dynamics, despite the simple form of $H$. Indeed one can interpret $U(g)$ as a potential and re-write the second of equations (3) as

$$
\frac{d \phi}{d t}=-\kappa^{D} d U(g)
$$

where $\phi=\phi_{a} d g^{a}$ is a one-form and $d U=\frac{\partial U}{\partial g^{a}} d g^{a}$ is the exterior derivative of the potential. The analogy between (4) and Newton's second law, $\frac{d p}{d t}=-d U$ for a particle with momentum $p$ moving in a potential $U$, is obvious.

The idea that RG flow might be related to a potential was first suggested, to the author's knowledge, by Wallace and Zia [4] but so far the investigations in this direction seem to have been attempts to find a potential for the $\beta$-functions which requires introducing 
a metric on the space of couplings, rather than for the VEV's as suggested here. In the construction presented here a metric on $T(\mathcal{M})$ is not necessary.

The reformulation of the RG evolution in terms of Hamiltonian flow allows the introduction of Poisson brackets and their associated symplectic structure. (That a symplectic structure should be relevant to RG flow was first suggested, to the authors knowledge, in [2].) Extending the set $\left\{g^{a}\right\}$ to include the cosmological constant, $\left\{g^{a}, \Lambda\right\}=\left\{g^{\hat{a}}\right\}$ where $\hat{a}=1, \ldots, n$, the Poisson bracket of any two quantities $A$ and $B$ is, of course, given by expressing them as functions of $g^{\hat{a}}$ and $\phi_{\hat{a}}$, possibly also with an explicit $t$ dependence, and taking the combination

$$
\{A, B\}=\frac{\partial A}{\partial g^{\hat{a}}} \frac{\partial B}{\partial \phi_{\hat{a}}}-\frac{\partial A}{\partial \phi_{\hat{a}}} \frac{\partial B}{\partial g^{\hat{a}}} .
$$

Obviously

$$
\left\{g^{\hat{a}}, \phi_{\hat{b}}\right\}=\delta_{\hat{a}}^{\hat{b}} \text {. }
$$

The RG evolution for any function on phase space is then given by

$$
\frac{d A}{d t}=\left.\frac{\partial A}{\partial t}\right|_{g, \phi}+\{A, H\}
$$

Note in particular that, when there is no explicit $\kappa$ dependence in the $\beta$-functions, the Hamiltonian (2) is a constant of the motion $\frac{d H}{d t}=0$. Once more we stress that, even though it is trivial that $\phi_{\Lambda}=\kappa^{D}$, it would be wrong to include this $\kappa$ dependence in $H(g, \phi)$ explicitly $-\phi_{\Lambda}$ is to be considered as an independent variable in this formalism and $H$ has no explicit $\kappa$ dependence. One could, however, omit $\Lambda$ as an independent variable and define a $t$-dependent Hamiltonian $h(g, \phi, t)=\beta^{a}(g) \phi_{a}+\kappa^{D} U(g)$ on $T^{*}(\mathcal{M})$. This leads to the same equations of motion $(3)$ but $h(g, \phi, t)$ is not a constant if there are massive couplings in the theory, instead $\frac{d h}{d t}=\frac{\partial h}{\partial t}=D \kappa^{D} U(g)$.

The classical analogy can be taken even further. It is also argued in section three that the RG equation for the generating function $w(g, t)$ can be written as

$$
\left.\frac{\partial w}{\partial t}\right|_{g}+H\left(g, \frac{\partial w}{\partial g}\right)=0
$$

which is clearly a field theoretic version of the Hamilton-Jacobi equation.

The layout of the paper will be as follows. In $\S 2$ the $\mathrm{RG}$ equation for $w(g, t)$ is derived, taking particular care over the role of the identity operator and the way that the cosmological constant is related to masses. In $\S 3$ a Hamiltonian formalism of RG evolution is developed. A symplectic structure on the phase space $\left(g^{\hat{a}}, \phi_{\hat{a}}\right)$ is introduced and Hamilton's equations (3) are derived together with the Hamilton-Jacobi equation (8). The renormalisation group equation for $N$-point Green functions is presented as a special case of equation $(7)$. $\S 4$ (which is the only part of this paper which uses perturbation theory) exemplifies the ideas with the use of massive $\lambda \varphi^{4}$ in four dimensions as a model. The implementation of symmetries is discussed in $\S 5$, where some examples are used to argue that Ward identities can be represented by symmetries on phase space and can be used to construct RG invariants ("constants of motion") which commute with the Hamiltonian and generate the symmetry through the Poisson bracket structure. Finally the results are summarised in $\S 6$. 


\section{$\S 2$ The Renormalisation Group Equation For The Partition Function}

Consider a renormalisable field theory, parameterised by a set of renormalised couplings $g_{R}^{a}, a=1, \ldots, n-1$ where $n$ is finite (the superscript $n$ will be reserved for a cosmological constant). In this section all renormalised quantities will be denoted by the letter $R$ so as to try to keep the argument as clear as possible. The bare couplings $g_{0}^{a}$ can be thought of as functions $g_{0}^{a}\left(g_{R}, \epsilon\right)$ of the renormalised couplings plus a regularisation parameter, $\epsilon$ (strictly speaking these functions should also contain an explicit $\kappa$ dependence since their total derivative with respect to $\kappa$ must vanish, but this is not shown here). The bare couplings are analytic functions of the $g_{R}^{a}$ provided $\epsilon \neq 0$, but are singular in the limit $\epsilon \rightarrow 0$. The transformation $g_{0}^{a} \rightarrow g_{R}^{a}$ can be viewed as a co-ordinate transformation on the $n$-1-dimensional space of theories, $\mathcal{M}$. This co-ordinate transformation is singular in the limit $\epsilon \rightarrow 0$ but, as long as the theory is renormalisable, this is not a disaster and can be treated consistently. For the moment the method of regularisation is left open, one could for example use a cut-off $\Lambda_{c}$ and set $\epsilon=\kappa / \Lambda_{c}$ or dimensional continuation with $\epsilon=D-4$.

The action can be written as a linear combination of "basic" operators $\Phi_{a}^{0}$, which will include composite operators,

$$
S_{0}\left(g_{0}, \Phi^{0}, \epsilon\right)=\int \mathcal{L}_{0}\left(g_{0}, \Phi^{0}(x)\right) d^{D} x \quad \text { where } \quad \mathcal{L}_{0}=g_{0}^{a} \Phi_{a}^{0}(x) .
$$

Strictly speaking, since space is taken to be Euclidean, this is the energy rather than the action - but a Hamiltonian will appear in the next section in a totally different context. By abuse of language therefore $S_{0}$ will be called the action.

In massive $\lambda \varphi^{4}$ theory, for example, one would have

$$
\mathcal{L}_{0}=k_{0} \partial_{\mu} \varphi_{0} \partial^{\mu} \varphi_{0}+j_{0} \varphi_{0}+\frac{1}{2} m_{0}^{2} \varphi_{0}^{2}+\frac{\lambda_{0}}{4 !} \varphi_{0}^{4}
$$

with $\Phi_{k}^{0}=\partial_{\mu} \varphi_{0} \partial^{\mu} \varphi_{0}, \Phi_{j}^{0}=\varphi_{0}, \Phi_{m^{2}}^{0}=\frac{1}{2} \varphi_{0}^{2}$ and $\Phi_{\lambda}^{0}=\frac{1}{4 !} \varphi_{0}^{4}$ and four independent couplings $k_{0}, j_{0}, m_{0}^{2}$ and $\lambda_{0}$. Without loss of generality the fields can be re-scaled to set $k_{0}=1$. If necessary the couplings can be made functions of position. Thus the notation can be extended to allow source terms, $j_{0}(x) \varphi_{0}(x)$, with $j_{0}(x)$ a function of position which may be set to zero after all (functional) differentiations have been carried out. More generally all of the couplings can be made to depend on position so as to introduce sources for the composite operators as well [5]. After all differentiations have been carried out these sources can be set to constant values if so desired.

Returning to the general case, consider a renormalisable theory written in terms of the bare couplings. Representing all of the bare fields generically by $\varphi_{0}$, the partition function (generating functional) is,

$$
Z_{0}\left(g_{0}\right)=\int \mathcal{D} \varphi_{0} \mathrm{e}^{-S_{0}\left(g_{0}, \Phi^{0}\right)} .
$$

The generating functional for connected Green functions (the free energy) is $W_{0}\left(g_{0}\right)=$ $-\ln Z_{0}\left(g_{0}\right)$. As usual the free energy density is defined via $W_{0}\left(g_{0}\right)=\int w_{0}\left(g_{0}, x\right) d^{D} x$. The 
introduction of $w_{0}$ avoids trivial volume divergences in the case of infinite space. It has canonical mass dimension $D$.

For future convenience a coupling for the identity operator will be included,

$$
Z\left(g_{0}, \Lambda_{0}\right)=\int \mathcal{D} \varphi_{0} \mathrm{e}^{-S_{0}\left(g_{0}, \Phi^{0}\right)-\int d^{D} x \Lambda_{0}}=\mathrm{e}^{-\int d^{D} x \Lambda_{0}} Z_{0}\left(g_{0}\right)
$$

(there is no bare subscript on $Z\left(g_{0}, \Lambda_{0}\right)$ because, as will be explained later, it is finite). In analogy with general relativity $\Lambda_{0}$ might be called a cosmological constant, but in different physical situations it would have different physical interpretations.

It is important to realise that $\Lambda_{0}$ is independent of $g_{0}^{a}$ and plays the role of a new coupling for the identity operator. Thus the set $\left\{g_{0}^{a}\right\}$ can be extended to $\left\{g_{0}^{\hat{a}}\right\}=\left\{g_{0}^{a}, \Lambda_{0}\right\}$ where $\hat{a}=1, \ldots, n$ are co-ordinates on a $n$-dimensional manifold $\widehat{\mathcal{M}} . \Lambda_{0}$ can be included in the bare Lagrangian as $\mathcal{L}_{0}=g_{0}^{a} \Phi_{a}^{0}+\Lambda_{0} \mathbf{1}$. In terms of densities

$$
W\left(g_{0}, \Lambda_{0}\right)=-\ln Z\left(g_{0}, \Lambda_{0}\right)=\int w\left(g_{0}, \Lambda_{0}\right) d^{D} x
$$

with $w\left(g_{0}, \Lambda_{0}\right)=w_{0}\left(g_{0}^{a}\right)+\Lambda_{0}$ linear in $\Lambda_{0}$.

When all couplings are independent of position and the theory is translationally invariant $w\left(g_{0}, \Lambda_{0}\right)$ is independent of $x$ and this will be assumed from now on. In situations in which translational invariance is not a symmetry of $D$-dimensional space, one will need to introduce extra terms involving the Riemann tensor into the action [3].

Expectation values of bare quantities can be obtained by differentiating $w\left(g_{0}, \Lambda_{0}\right)$ with respect to the couplings,

$$
<\Phi_{a}^{0}>=\left\langle\frac{\partial \mathcal{L}_{0}}{\partial g_{0}^{a}}\right\rangle=\frac{\partial w}{\partial g_{0}^{a}} \quad \text { and } \quad 1=<\mathbf{1}>=\frac{\partial w}{\partial \Lambda_{0}} .
$$

The bare operators $\Phi_{\hat{a}}^{0}=\frac{\partial \mathcal{L}_{0}}{\partial g_{0}^{\hat{a}}}$ are co-vectors on the space of couplings, i.e. $\Phi:=\Phi_{\hat{a}}^{0} d g_{0}^{\hat{a}}$ is an operator valued one-form. This notion of the basic operators being co-vectors on the space of couplings is implicit in the work of Zamolodchikov [6]. For a conformal field theory in two dimensions $\Phi_{\hat{a}}^{0}$ would be the primary fields of the theory. Expectation values, $d w=<\Phi_{\hat{a}}^{0}>d g_{0}^{\hat{a}}$, are (exact) real valued one-forms on $\widehat{\mathcal{M}}$.

The bare operators can be written as linear combinations of renormalised operators

$$
\Phi_{\hat{a}}^{0}=Z_{\hat{a}}^{\hat{b}} \Phi_{\hat{b}}^{R}
$$

where $Z_{\hat{a}}^{\hat{b}}$ is a matrix of renormalisation constants (see for example [7]). In general there will be operator mixing and $Z_{\hat{a}}^{\hat{b}}$ will not be diagonal. If the bare action has no massive couplings it is not necessary to include the identity operator in the list and a cosmological constant can be omitted, since $Z_{a}{ }^{n}=0(a \leq n-1)$ when all couplings are massless. But if there are massive couplings in the bare action then some or all of the $Z_{a}{ }^{n}$ will be non-zero and $\Lambda_{0}$ plays a crucial role. 
Following up the idea that the transition from bare to renormalised couplings can be implemented as a co-ordinate transformation on $\widehat{\mathcal{M}}$, we consider $g_{0}^{\hat{a}}\left(g_{R}, \epsilon\right)$ to be analytic functions of $g_{R}^{\hat{a}}$ (keeping $\epsilon \neq 0$ for the moment). Consider

$$
\frac{\partial \mathcal{L}_{0}}{\partial g_{R}^{\hat{a}}}=\frac{\partial g_{0}^{\hat{b}}}{\partial g_{R}^{\hat{a}}} \frac{\partial \mathcal{L}_{0}}{\partial g_{0}^{\hat{b}}}=\frac{\partial g_{0}^{\hat{b}}}{\partial g_{R}^{\hat{a}}} \Phi_{\hat{b}}^{0}
$$

Clearly these should be related to the renormalised operators $\Phi_{\hat{a}}^{R}$. In fact

$$
\frac{\partial g_{0}^{\hat{b}}}{\partial g_{R}^{\hat{a}}}=\left(Z^{-1}\right)_{\hat{a}}^{\hat{b}}
$$

is the inverse of the operator mixing matrix $Z_{\hat{a}}^{\hat{b}}=\frac{\partial g_{R}^{\hat{b}}}{\partial g_{0}^{\hat{a}}}[8]$. Thus

$$
\frac{\partial \mathcal{L}_{0}}{\partial g_{R}^{\hat{a}}}=\left(Z^{-1}\right)_{\hat{a}}^{\hat{b}} \Phi_{\hat{b}}^{0}=\Phi_{\hat{a}}^{R}
$$

The operator valued one-form $\Phi$ can now be expressed in either co-ordinate system,

$$
\Phi=\Phi_{\hat{a}}^{0} d g_{0}^{\hat{a}}=\Phi_{\hat{a}}^{R} d g_{R}^{\hat{a}}
$$

and similarly the real valued one-form $d w$ is

$$
d w=<\Phi>=<\Phi_{\hat{a}}^{0}>d g_{0}^{\hat{a}}=<\Phi_{\hat{a}}^{R}>d g_{R}^{\hat{a}}
$$

Thus

$$
\frac{\partial w}{\partial g_{R}^{\hat{a}}}=\frac{\partial g_{0}^{\hat{b}}}{\partial g_{R}^{\hat{a}}}<\Phi_{\hat{b}}^{0}>=\left(Z^{-1}\right)_{\hat{a}}^{\hat{b}}<\Phi_{\hat{b}}^{0}>=<\Phi_{\hat{a}}^{R}>.
$$

Partial derivatives here mean, of course, that all quantities are considered to be functions of the renormalised couplings, and each $g_{R}^{\hat{a}}$ is varied independently of the others. When massive couplings are present $\Lambda_{0}$ must be considered to be a function of the renormalised couplings. This is necessary because the $\Phi_{0}^{a}$ then mix with the identity operator under renormalisation.

Note that, since both $g_{R}^{\hat{a}}$ and $\left\langle\Phi_{\hat{a}}^{R}\right\rangle$ in equation (21) are finite as the regularisation parameter $\epsilon \rightarrow 0, w\left(g_{R}, t\right)$ considered as a function of the renormalised couplings and the renormalisation point $\kappa$ must also be finite as $\epsilon \rightarrow 0$. This is why there is no bare subscript on the definition of $Z\left(g_{0}, \Lambda_{0}\right)$ in equation (12), [3], and is one of the reasons for introducing a coupling for the identity operator - both $Z_{0}\left(g_{0}^{a}\right)$ and $\Lambda_{0}$ seperately diverge as $\epsilon \rightarrow 0$ but the combination $Z\left(g_{R}^{\hat{a}}\right)=Z\left(g_{0}^{\hat{a}}\right)=\mathrm{e}^{-\int d^{D} x \Lambda_{0}} Z_{0}\left(g_{0}^{a}\right)$ is finite. A finite, renormalised generating function, $W_{R}\left(g_{R}^{a}, t\right)=\int w_{R}\left(g_{R}^{a}, t\right) d^{D} x=-\ln Z_{R}\left(g_{R}^{a}\right)$, can now be defined by

$$
Z\left(g_{0}^{\hat{a}}\right)=\mathrm{e}^{-\int d^{D} x \Lambda_{0}} Z_{0}\left(g_{0}^{a}\right)=Z\left(g_{R}^{\hat{a}}, t\right)=\mathrm{e}^{-\kappa^{D} \int d^{D} x \Lambda_{R}} Z_{R}\left(g_{R}^{a}, t\right)
$$


(the factor $\kappa^{D}$ in the exponential is in accord with the convention that $\Lambda_{R}$ is dimensionless). Thus $w=w_{0}\left(g_{0}^{a}\right)+\Lambda_{0}=w_{R}\left(g_{R}^{a}, t\right)+\Lambda_{R} \kappa^{D}$ can now be interpreted as a finite function of the renormalised couplings with the crucial property that

$$
\frac{\partial w}{\partial g_{R}^{a}}=<\Phi_{a}^{R}>\quad \text { and } \quad \frac{\partial w}{\partial \Lambda_{R}}=<\Phi_{n}^{R}>=<\kappa^{D} \mathbf{1}>=\kappa^{D} .
$$

Note that $\Lambda_{0}$ is linear in $\Lambda_{R}$, a fact which follows from the observation that the identity operator $\mathbf{1}=\Phi_{n}^{0}$ does not get renormalised. Thus

$$
\left(Z^{-1}\right)_{\hat{a}}^{\hat{b}}=\left(\begin{array}{cc}
\left(Z^{-1}\right)_{a}{ }^{b} & \left(Z^{-1}\right)_{a}{ }^{n} \\
0 & \kappa^{D}
\end{array}\right) \quad \text { since } \quad \frac{\partial g_{0}^{n}}{\partial g_{R}^{n}}=\frac{\partial \Lambda_{0}}{\partial \Lambda_{R}}=\kappa^{D} .
$$

Indeed even the $\beta$-function for $\Lambda_{R}$ only depends on $\Lambda_{R}$ linearly through canonical dimensions, since writing

$$
\Lambda_{0}=\kappa^{D}\left(\Lambda_{R}+F\left(g_{R}^{a}, \epsilon\right)\right)
$$

we have

$$
\beta^{\Lambda}=\frac{d \Lambda_{R}}{d t}=-D \Lambda_{R}+U\left(g_{R}^{a}\right)
$$

where $U\left(g_{R}^{a}\right):=-\frac{d F}{d t}$ depends only on the $g_{R}^{a}$ for $a \leq n-1$, not on $\Lambda_{R}$, and is finite as $\epsilon \rightarrow 0$. The fact that $U\left(g_{R}\right)$ is independent of $\Lambda$ can be seen from the following argument. $U\left(g_{R}\right)$ is a quantum correction to the canonical dimensions of the cosmological constant and as such can be determined (in principle) using perturbation theory and Feynman diagrams, but $\Lambda$ cancels out of all Feynamn diagrams due to the normalisation factor $\frac{1}{z}$. Hence $U\left(g_{R}\right)$ is independent of $\Lambda$.

To make contact with the familiar notions of a perturbative analysis one can write the bare couplings in terms of the renormalised couplings as

$$
g_{0}^{\hat{a}}\left(g_{R}, \epsilon\right)=g_{R}^{\hat{a}}+\Delta g^{\hat{a}}\left(g_{R}, \epsilon\right)
$$

where $\Delta g^{\hat{a}}$ is a correction (which diverges as $\epsilon \rightarrow 0$ ), so that

$$
\begin{aligned}
\mathcal{L}_{0} & =g_{0}^{\hat{a}} \Phi_{\hat{a}}^{0}=\left(g_{R}^{\hat{a}}+\Delta g^{\hat{a}}\right) Z_{\hat{a}}^{\hat{b}} \Phi_{\hat{b}}^{R} \\
& =g_{R}^{\hat{a}} \Phi_{\hat{a}}^{R}+\text { counter terms } \quad \text { (C.T.'s). }
\end{aligned}
$$

This gives

$$
\mathrm{e}^{-W}=Z\left(g_{0}, \Lambda_{0}\right)=\mathrm{e}^{-\int d^{D} x \Lambda_{0}} \int \mathcal{D} \varphi_{0} \mathrm{e}^{-\int d^{D} x g_{R}^{a} \Phi_{a}^{R}(x)+C . T{ }^{\prime} s},
$$

where the identity operator has been included among the counter terms. Absorbing a further term proportional to the identity into the counter terms we have

$$
\mathrm{e}^{-W}=\mathrm{e}^{-\kappa^{D} \int d^{D} x \Lambda_{R}} \int \mathcal{D} \varphi_{0} \mathrm{e}^{-\int d^{D} x g_{R}^{a} \Phi_{a}^{R}(x)+C . T .^{\prime} s} .
$$


If desired, one can perform the functional integral over renormalised fields rather than bare fields by setting $\mathcal{D} \varphi_{0} \rightarrow \mathcal{D} \varphi_{R}$, where $\varphi_{0}=z^{1 / 2} \varphi_{R}$ with $z$ being the wave function renormalisation factor, and then adding a further term $\frac{1}{2} \ln z \int d^{D} x \mathbf{1}$ to the counter terms.

Leaving perturbation theory behind and returning to the general analysis, we are now in a position to write the renormalisation group equation for the free energy, $w\left(g_{R}^{\hat{a}}, t\right)$. Since $w=w_{0}\left(g_{0}^{a}\right)+\Lambda_{0}$ and all bare couplings are independent of the renormalisation point $t=\ln \kappa$, we have

$$
\frac{d w\left(g_{R}^{a}, \Lambda_{R}, t\right)}{d t}=\beta^{a} \frac{\partial w}{\partial g_{R}^{a}}+\beta^{\Lambda} \frac{\partial w}{\partial \Lambda_{R}}+\left.\frac{\partial w}{\partial t}\right|_{g_{R}, \Lambda_{R}}=0
$$

If we now denote the VEV's by $\phi_{a}^{R}:=<\Phi_{a}^{R}>$, this reads (since $\frac{\partial w}{\partial \Lambda_{R}}=\kappa^{D}=\phi_{\Lambda}^{R}$ and $\left.\frac{\partial w}{\partial g_{R}^{a}}=\phi_{a}^{R}\right)$

$$
\left.\frac{\partial w\left(g_{R}^{\hat{a}}, t\right)}{\partial t}\right|_{g_{R}, \Lambda_{R}}+\beta^{a}\left(g_{R}^{a}\right) \phi_{a}^{R}+\beta^{\Lambda}\left(g_{R}^{\hat{a}}\right) \phi_{\Lambda}=0 .
$$

This is the equation that will be used in the next section to argue for Hamiltonian flow on $\left(g_{R}^{\hat{a}}, \phi_{\hat{a}}^{R}\right)$ space.

Alternatively, since $\Lambda_{R}$ only ever appears in this equation linearly, it can be eliminated using equation (26) and $w=w_{R}\left(g_{R}^{a}\right)+\Lambda_{R} \kappa^{D}$, with $w_{R}$ independent of $\Lambda_{R}$, to give

$$
\left.\frac{\partial w_{R}\left(g_{R}^{a}, t\right)}{\partial t}\right|_{g_{R}}+\beta^{a}\left(g_{R}^{a}\right) \phi_{a}^{R}+\kappa^{D} U\left(g_{R}^{a}\right)=0 .
$$

Note that it is not true, in general, that $\frac{d w_{R}\left(g_{R}^{a}, t\right)}{d t}=0$, since the presence of massive couplings necessitates the introduction the function $U(g)$ in a general renormalisation prescription.

This analysis has been a somewhat lengthy treatment of concepts that are not new, but it has been included in order to expose clearly the role of the cosmological constant in theories with massive couplings as well as to set up the notation. 


\section{$\S 3$ Symplectic Structure And Hamiltonian Flow Of The RG Equation}

In this section it will be shown that the RG equation derived for the generating function in the previous section naturally admits a symplectic structure with its concomitant Poisson brackets, and the renormalisation group flow can be obtained from a Hamiltonian function on phase space in a manner analogous to dynamical evolution in classical mechanics (but with important differences).

The starting point is equation (32)

$$
\phi_{\hat{a}} \frac{d g^{\hat{a}}}{d t}+\left(\frac{\partial w}{\partial t}\right)=0 .
$$

From now on the qualifier $R$ on $g_{R}^{\hat{a}}$ and $\phi_{\hat{a}}^{R}$ will be omitted as all quantities will be renormalised, unless otherwise indicated. The generating function $w$ appearing in this section is always $w=w_{R}+\kappa^{D} \Lambda_{R}$. It is stressed that $w$ is linear in $\Lambda$.

To highlight the analogy with classical mechanics, we shall define a function $H$,

$$
H=-\frac{\partial w}{\partial t},
$$

so that (34) can be written

$$
H(g, \phi)=\beta^{a}(g) \phi_{a}+\beta^{\Lambda} \phi_{\Lambda} .
$$

(For the moment it will be assumed that the $\beta$-functions have no explicit $\kappa$ dependence so that $H(g, \phi)$ has no explicit $t$ dependence - a generalisation including explicitly $\kappa$ dependent $\beta$-functions is given towards the end of this section.) The philosophy now is to forget where the $\phi_{\hat{a}}$ came from and treat them as independent variables. It is only after the theory has been solved that we can use $\phi_{\hat{a}}=\partial_{\hat{a}} w$.

Consider the left hand side of equation (34) as a differential

$$
\Theta=\phi_{\hat{a}} d g^{\hat{a}}-H d t,
$$

where $\Theta(g, \phi, t)$ is a one-form on the $2 n+1$ dimensional space parameterised by $g^{\hat{a}}, \phi_{\hat{a}}$ and $t$. When the theory is solved, and $\phi_{\hat{a}}(g, t)$ is written as an explicit function of $g^{\hat{a}}$ and $t$, $\Theta=d w$ is exact, but when the $\phi_{\hat{a}}$ are treated as independent variables $\Theta$ is not exact. The discussion now parallel's the treatment of classical mechanics in [9]. Just as the couplings $g^{\hat{a}}(t)$ evolve along the RG trajectories, so do the expectation values $\phi_{\hat{a}}(t)$. Thus the RG trajectories can be pictured as flow lines in $\left(g^{\hat{a}}, \phi_{\hat{a}}, t\right)$ space. Treating $\phi_{\hat{a}}$ as independent variables construct the two-form

$$
\begin{aligned}
\Omega & =\frac{1}{2} \Omega_{i j} d x^{i} \wedge d x^{j}=d \Theta=d \phi_{\hat{a}} \wedge d g^{\hat{a}}-d H \wedge d t \\
& =d \phi_{\hat{a}} \wedge d g^{\hat{a}}-\left.\frac{\partial H}{\partial g^{\hat{a}}}\right|_{\phi} d g^{\hat{a}} \wedge d t-\left.\frac{\partial H}{\partial \phi_{\hat{a}}}\right|_{g} d \phi_{\hat{a}} \wedge d t,
\end{aligned}
$$


where $\left\{x^{i}\right\}=\left\{g^{\hat{a}}, \phi_{\hat{a}}, t\right\} i=1, \ldots, 2 n+1$ are co-ordinates on the $2 n+1$ dimensional space. Now $\Omega$ can be written as an anti-symmetric matrix,

$$
\Omega_{i j}=\left(\begin{array}{ccc}
0 & -\mathbf{I} & -\left.\frac{\partial H}{\partial g^{\hat{a}}}\right|_{\phi} \\
\mathbf{I} & 0 & -\left.\frac{\partial H}{\partial \phi_{\hat{a}}}\right|_{g} \\
\left.\frac{\partial H}{\partial g^{\hat{a}}}\right|_{\phi} & \left.\frac{\partial H}{\partial \phi_{\hat{a}}}\right|_{g} & 0
\end{array}\right)
$$

where $\mathbf{I}$ is the $n \times n$ identity matrix. Since $\Omega_{i j}$ is an odd dimensional anti-symmetric matrix it must have at least one zero eigenvalue (it will be assumed that it has only one, otherwise the restriction of $\Omega$ to surfaces of constant $t$ would result in a degenerate symplectic form on phase space). The corresponding eigenvector, $\vec{\xi}$, is easily seen to be

$$
\xi^{i}=\left(\left.\frac{\partial H}{\partial \phi_{\hat{a}}}\right|_{g},-\left.\frac{\partial H}{\partial g^{\hat{a}}}\right|_{\phi}, 1\right) .
$$

It seems natural to identify the flow lines of the vector field $\vec{\xi}$ with renormalisation group trajectories, since $\Theta$ is exact when the theory is solved and $\phi_{\hat{a}}(g, t)$ are substituted into equation (37). This requires

$$
\frac{d g^{\hat{a}}}{d t}=\left.\frac{\partial H}{\partial \phi_{\hat{a}}}\right|_{g} \quad \text { and } \quad \frac{d \phi_{\hat{a}}}{d t}=-\left.\frac{\partial H}{\partial g^{\hat{a}}}\right|_{\phi} .
$$

Obviously $\left.\frac{\partial H}{\partial \phi_{\hat{a}}}\right|_{g}=\beta^{\hat{a}}$ by definition, so the first equation is certainly consistent. For $\hat{a}=n$, the second equation reduces to the identity $\frac{d \kappa^{D}}{d t}=D \kappa^{D}$. The interpretation of the other equation hinges on the crucial observation that the function $U(g)=\beta^{\Lambda}+D \Lambda$ is an analytic function of the $g^{a}$ (independent of $\Lambda$ ). Thus, from equation (36)

$$
\frac{d \phi_{a}}{d t}=-\frac{\partial}{\partial g^{a}}\left(\kappa^{D} U(g)\right)-\left(\frac{\partial \beta^{b}}{\partial g^{a}}\right) \phi_{b}
$$

or

$$
\frac{d \phi_{a}}{d t}+\left(\partial_{a} \beta^{b}\right) \phi_{b}=-\kappa^{D} \partial_{a} U
$$

This is the renormalisation group equation for the $R G$ evolution of the vacuum expectation values of the basic operators of the theory. The parallel with Newton's second law is obvious. The matrix of anomalous dimensions $\partial_{a} \beta^{b}$ appears as a pseudo-force (Coriolis force) and the function $U(g)$ is a potential. For massless theories $U$ vanishes, thus massless theories are analogous to free particle motion. Just as in classical mechanics the Coriolis force can be eliminated when the motion of the basis vectors is included in the equation. Consider therefore the one-form $\phi=\phi_{a} d g^{a}$. One has

$$
\begin{aligned}
\frac{d \phi}{d t} & =\left(\frac{d \phi_{a}}{d t}\right) d g^{a}+\phi_{a} \frac{d\left(d g^{a}\right)}{d t} \\
& =\left(\frac{d \phi_{a}}{d t}+\phi_{b} \frac{\partial \beta^{b}}{\partial g^{a}}\right) d g^{a}
\end{aligned}
$$


where we have used $\frac{d\left(d g^{a}\right)}{d t}=d\left(\frac{d g^{a}}{d t}\right)=\partial_{b} \beta^{a} d g^{b}$. Thus equation (43) can be written in co-ordinate free notation as,

$$
\frac{d \phi}{d t}=-\kappa^{D} d U(g)
$$

where $d U=\frac{\partial U}{\partial g^{a}} d g^{a}$ is the exterior derivative. Of course, once the theory is solved, the $\phi_{a}$ can be expressed as explicit functions of $g^{a}$ and $t, \phi_{a}(g, t)$, so that

$$
\frac{d \phi}{d t}=\left(\left.\frac{\partial \phi_{a}}{\partial t}\right|_{g}+\beta^{b} \partial_{b} \phi_{a}+\left(\partial_{a} \beta^{b}\right) \phi_{b}\right) d g^{a}
$$

and equation (43) then becomes

$$
\left.\frac{\partial \phi_{a}}{\partial t}\right|_{g}+\beta^{b} \partial_{b} \phi_{a}+\left(\partial_{a} \beta^{b}\right) \phi_{b}=-\kappa^{D} \partial_{a} U
$$

which is a version of the RG equation for the VEV's, including the anomalous dimensions and the inhomogeneous term $-\kappa^{D} \partial_{a} U$ which arises due to masses.

Yet another way of expressing this is to observe that the left hand side of (46) involves the Lie derivative, $\mathcal{L}_{\vec{\beta}} \phi$, of the one-form $\phi_{a} d g^{a}$ with respect to the vector field $\vec{\beta}=\beta^{a} \frac{\partial}{\partial g^{a}}$ $[10]$. Since $\phi$ is exact we have

$$
\mathcal{L}_{\vec{\beta}} \phi=d\left(i_{\vec{\beta}} \phi\right)=\frac{\partial}{\partial g^{a}}\left\{\beta^{b}(g) \phi_{b}(g, t)\right\} d g^{a}
$$

where $i_{\vec{\beta}} \phi$ denotes the contraction of $\vec{\beta}$ with the one-form $\phi, i_{\vec{\beta}} \phi=\beta^{a} \phi_{a}$. Thus another way of writing (43) is

$$
\left.\frac{\partial \phi}{\partial t}\right|_{g}=-d\left\{\kappa^{D} U(g)+\beta^{b}(g) \phi_{b}(g)\right\},
$$

where again $d=d g^{a} \frac{\partial}{\partial g^{a}}$.

The analogy with classical mechanics can be taken further still. The definition of $H$ in equation (35),

$$
H(g, \phi)+\frac{\partial w}{\partial t}=0
$$

can be expressed as a partial differential equation in the $n+1$ variables $\left(g^{\hat{a}}, t\right)$. Since, when the theory is solved, $\phi_{\hat{a}}=\frac{\partial w}{\partial g^{\hat{a}}}$ we have

$$
\left.\frac{\partial w}{\partial t}\right|_{g}+H\left(g, \frac{\partial w}{\partial g}\right)=0
$$

which is clearly an analogue of the Hamilton-Jacobi equation. (That the RG equation ought to be expressable as a Hamilton-Jacobi type equation was first suggested to the author by Denjoe O'Connor and Chris Stephens [11] .) Thus the generating functional in quantum field theory (or free energy density in statistical mechanics) is playing the role of the action in classical mechanics (Hamilton's principal function). 
This structure suggests a reformulation of the renormalisation group. Instead of expressing the RG running in terms of $\beta$-functions and couplings, which can be thought of as co-ordinates on configuration space (the tangent bundle $T(\widehat{\mathcal{M}})$ ) it may be useful to use instead phase space variables (the co-tangent bundle $T^{*}(\widehat{\mathcal{M}})$ ). Any quantity, $A$, should then be considered to be a function of the $2 n$ co-ordinates $\left(g^{\hat{a}}, \phi_{\hat{a}}\right)$ and possibly also the renormalisation point $t=\ln \kappa$. The $\mathrm{RG}$ evolution of $A(g, \phi, t)$ is then given by,

$$
\frac{d A}{d t}=\frac{\partial A}{\partial g^{\hat{a}}} \frac{\partial H}{\partial \phi_{\hat{a}}}-\frac{\partial A}{\partial \phi_{\hat{a}}} \frac{\partial H}{\partial g^{\hat{a}}}+\left.\frac{\partial A}{\partial t}\right|_{g, \phi}=\{A, H\}+\left.\frac{\partial A}{\partial t}\right|_{g, \phi},
$$

where $\{A, H\}$ is the usual Poisson bracket with $\left\{g^{\hat{a}}, \phi_{\hat{b}}\right\}=\delta^{\hat{a}}{ }_{\hat{b}}$. Since there is no explicit $\kappa$ dependence in the Hamiltonian $(36) H(g, \phi)$ is a RG invariant (a constant of the motion)

$$
\frac{d H}{d t}=0
$$

but this is only true when there is no explicit $\kappa$ dependence in the $\beta$-functions.

In particular the RG evolution of $N$-point Green functions is of key importance in any theory. These can be viewed as rank $N$ tensors on the space of couplings,

$$
G_{a_{1} \cdots a_{N}}^{(N)}\left(x_{1}, \ldots, x_{N}\right)=<\tilde{\Phi}_{a_{1}}\left(x_{1}\right) \cdots \tilde{\Phi}_{a_{N}}\left(x_{N}\right)>
$$

where $\tilde{\Phi}_{a_{i}}\left(x_{i}\right)=\Phi_{a_{i}}\left(x_{i}\right)-\phi_{a_{i}}$ has zero vacuum expectation value (and is independent of $\Lambda)$. The RG equation for $G_{a_{1} \cdots a_{N}}^{(N)}\left(x_{1}, \ldots, x_{N}\right)$ is obtained by the process described above,

$$
\begin{aligned}
& \frac{d}{d t} G_{a_{1} \cdots a_{N}}^{(N)}\left(x_{1}, \ldots, x_{N}\right)= \\
& \quad\left(\left.\frac{\partial}{\partial t}\right|_{g, \phi}+\left.\beta^{b} \frac{\partial}{\partial g^{b}}\right|_{\phi, t}-\left.\phi_{c}\left(\frac{\partial \beta^{c}}{\partial g^{b}}\right) \frac{\partial}{\partial \phi_{b}}\right|_{g, t}-\left.\kappa^{D} \partial_{b} U \frac{\partial}{\partial \phi_{b}}\right|_{g, t}\right) G_{a_{1} \cdots a_{N}}^{(N)}\left(x_{1}, \ldots, x_{N}\right) .
\end{aligned}
$$

If the co-vector basis $d g^{a}$ is also included, so as to write the tensor in co-ordinate free notation $G^{(N)}=G_{a_{1} \cdots a_{N}}^{(N)}\left(x_{1}, \ldots, x_{N}\right) d g^{a_{1}} \cdots d g^{a_{N}}$, one arrives at the equation

$$
\begin{aligned}
\left(\frac{d G^{(N)}}{d t}\right)_{a_{1} \cdots a_{N}}=\frac{\partial}{\partial t} G_{a_{1} \cdots a_{N}}^{(N)} & +\left.\beta^{b} \frac{\partial}{\partial g^{b}}\right|_{\phi, t} G_{a_{1} \cdots a_{N}}^{(N)}+\sum_{i=1}^{N}\left(\partial_{a_{i}} \beta^{b}\right) G_{a_{1} \cdots a_{i-1} b a_{i+1} \cdots a_{N}}^{(N)} \\
& -\left.\phi_{c}\left(\partial_{b} \beta^{c}\right) \frac{\partial}{\partial \phi_{b}}\right|_{g, t} G_{a_{1} \cdots a_{N}}^{(N)}-\left.\kappa^{D} \partial_{b} U \frac{\partial}{\partial \phi_{b}}\right|_{g, t} G_{a_{1} \cdots a_{N}}^{(N)} .
\end{aligned}
$$

The RG equation for $N$-point Green functions in the Hamiltonian formalism is finally obtained by observing that $G^{(N)}$ can equally well be written in bare co-ordinates and so is independent of $t$. The left hand side of (54) therefore vanishes, $\frac{d G^{(N)}}{d t}=0$, and the RG equation is

$$
\begin{aligned}
\frac{\partial}{\partial t} G_{a_{1} \cdots a_{N}}^{(N)}(g, \phi, t) & +\beta^{b} \partial_{b} G_{a_{1} \cdots a_{N}}^{(N)}(g, \phi, t)+\sum_{i=1}^{N}\left(\partial_{a_{i}} \beta^{b}\right) G_{a_{1} \cdots a_{i-1} b a_{i+1} \cdots a_{N}}^{(N)}(g, \phi, t) \\
& =\left(\phi_{c}\left(\partial_{b} \beta^{c}\right)+\kappa^{D} \partial_{b} U\right) \frac{\partial}{\partial \phi_{b}} G_{a_{1} \cdots a_{N}}^{(N)}(g, \phi, t) .
\end{aligned}
$$


Alternatively, using equation (46) and allowing for the $g^{a}$ and $t$ dependence of $\phi_{a}$ after the theory has been solved, this equation can be re-expressed as

$$
\begin{aligned}
& \frac{\partial}{\partial t} G_{a_{1} \cdots a_{N}}^{(N)}(g, \phi, t)+\beta^{b} \frac{\partial}{\partial g^{b}} G_{a_{1} \cdots a_{N}}^{(N)}(g, \phi, t)+\beta^{c}\left(\frac{\partial \phi_{b}(g, t)}{\partial g^{c}}\right) \frac{\partial}{\partial \phi_{b}} G_{a_{1} \cdots a_{N}}^{(N)}(g, \phi, t) \\
& \quad+\sum_{i=1}^{N}\left(\partial_{a_{i}} \beta^{b}\right) G_{a_{1} \cdots a_{i-1} b a_{i+1} \cdots a_{N}}^{(N)}(g, \phi, t)+\left(\frac{\partial \phi_{b}(g, t)}{\partial t}\right) \frac{\partial}{\partial \phi_{b}} G_{a_{1} \cdots a_{N}}^{(N)}(g, \phi, t)=0 .
\end{aligned}
$$

Were it not for the last term on the left hand side of this equation, it would just be the definition of the Lie derivative of $G^{(N)}$ with respect to the vector field $\vec{\beta}$ - the last term is a correction to this interpretation. That the RG equation for $N$-point amplitudes could be written as a Lie derivative was observed in [10], and corrections to this interpretation were investigated in [12] .

Returning now to the general formalism let us consider more general canonical transformations. A renormalisation group transformation is like time evolution in classical mechanics and as such preserves the symplectic structure on the $2 n$-dimensional space $\left(g^{\hat{a}}, \phi_{\hat{a}}\right)$,

$$
\widehat{\omega}=\left.\Omega\right|_{t=\mathrm{const}}=d \phi_{\hat{a}} \wedge d g^{\hat{a}} .
$$

Of course, there will in general be other important canonical transformations which do not necessarily correspond to RG transformations. In particular, the transformation from bare to renormalised couplings preserves $\widehat{\omega}$,

$$
\widehat{\omega}=d \phi_{\hat{a}} \wedge d g^{\hat{a}}=d \phi_{\hat{a}}^{0} \wedge d g_{0}^{\hat{a}}
$$

but in a general renormalisation prescription this would not correspond to a RG transformation (except perhaps if the theory is regularised by using a cut-off and BPHZ renormalisation, in which case the bare couplings do have the interpretation of just being the renormalised couplings at some very high energy, $\Lambda_{c}$ ). Since the bare couplings are independent of the renormalisation point, $\frac{d g_{0}^{\hat{a}}}{d t}=\frac{d \phi_{\hat{a}}^{0}}{d t}=0$, this transformation is analogous to the canonical transformation in classical mechanics which takes one from time dependent phase space variables $\left(q^{a}(t), p_{a}(t)\right)$ to the initial point $\left(q_{0}^{a}, p_{a}^{0}\right)$. Referring back to the Hamilton-Jacobi equation (8) we see that the generating function for this canonical transformation, in the familiar classical mechanical sense, is nothing other than the generating function(al) of the quantum field theory, $w$.

The analogy with the Hamilton-Jacobi equation of classical mechanics can be further highlighted by explicitly indicating that $w(g, t)$ depends on the subtraction procedure and writing it as $w\left(g(t), g_{0}, t\right)$. This emphasises its dependence on the counterterms $\Delta g^{\hat{a}}=g_{0}^{\hat{a}}-$ $g^{\hat{a}}(t)$, and does not affect the argument that $\frac{d w}{d t}=0$. In BPHZ renormalisation $g_{0}^{\hat{a}}$ really can be thought as lying on on the RG trajectory and the analogy between $w\left(g(t), g_{0}, t\right)$ and Hamilton's principal function, the action $S\left(q, q_{0}, t\right)$ along a classical trajectory is even stronger.

A crucial difference between the phase space approach to the RG presented here and classical mechanics lies in the Legendre transform,

$$
H(g, \phi)-\beta^{\hat{a}} \phi_{\hat{a}}=0,
$$


with $\beta^{\hat{a}}=\left.\frac{\partial H}{\partial \phi_{\hat{a}}}\right|_{g}$, which vanishes and in particular is not invertible. However, as the examples of the next two sections show, the flow is still far from trivial!

The Legendre transform presented here is also much simpler in form than quantum field theory Legendre transform introduced by Jona-Lasinio [13] .* The latter is implemented at the level of the generating functional $w$ itself, rather than on its derivative $\frac{\partial w}{\partial t}$, and this leads to the effective action, which most certainly does not vanish.

Note that the Hamiltonian (36),

$$
H=\beta^{a} \phi_{a}+\beta^{\Lambda} \phi_{\Lambda},
$$

actually has a simple physical interpretation. The right hand side of this equation is just the negative of the usual definition of the vacuum expectation value of the trace of the energy-momentum tensor of the theory, $H=-\langle T\rangle$. It should not come as a surprise that $\langle T\rangle=\left.\frac{\partial w}{\partial t}\right|_{g}$ since varying $t$ with the couplings fixed is completely equivalent to a conformal rescaling of the metric. The derivative $\left.\frac{\partial}{\partial t}\right|_{g}$ acting on $w$ simply pulls down the action from the exponent and then varies the metric leading to $\left\langle T_{\mu}^{\mu}\right\rangle$. Thus the entire $R G$ evolution is governed solely by $<T>$.

At fixed points of the RG flow (conformal field theories) the Hamiltonian vanishes, because the $\beta$-functions do. However once the theory is solved and explicit expressions for $\phi_{a}$ in terms of $g^{a}(t)$ and $t$ are substituted into the Hamiltonian, the resulting function is not analytic at fixed points. Derivatives higher than the first may be singular, as the example of $\lambda \varphi^{4}$ treated in the next section shows. This is to be expected since the Hamiltonain is defined in terms of the free energy density which is non-analytic at critical points.

The analysis so far has assumed that a subtraction procedure is chosen so that the $\beta$-functions only depend on $\kappa$ implicitly through $g^{\hat{a}}(\kappa)$ and have no explicit $\kappa$ dependence. Sometimes, however, it may be convenient to use a subtraction procedure which results in $\beta$-functions which have an explicit $\kappa$ dependence, $\beta^{\hat{a}}(g, t)$. This can be incorporated into the present framework by considering $t$ to be like an extra coupling and extending the $n$-dimensional manifold $\widehat{\mathcal{M}}$ to a $n+1$-dimensional manifold $\widehat{\mathcal{M}}_{E}$ with $t$ as the extra co-ordinate. The momentum conjugate to $t$ is (minus) the Hamiltonian, $\phi_{t}=\partial_{t} w=-H(g, \phi, t)$. The $2 n$-dimensional phase space $T^{*}(\widehat{\mathcal{M}})$ is now extended to a $2 n+2$-dimensional phase space $T^{*}\left(\widehat{\mathcal{M}}_{E}\right)$. By definition one has $\beta^{t}=1$. This is clearly analogous to the situation in classical mechanics where phase space is extended to include the energy and time as extra co-ordinates. The Hamiltonian on the extended phase space is

$$
\mathcal{H}_{E}(g, \phi, t)=\beta^{\hat{a}}(g, t) \phi_{\hat{a}}+\phi_{t},
$$

and a new evolution parameter $\tau$ is introduced which is ultimately identified with $t$ when the theory is solved. When the theory is solved one has $\mathcal{H}_{E}=0$, which is just the Hamilton-Jacobi equation for $H(g, \phi, t)=\beta^{\hat{a}} \phi_{\hat{a}}$,

$$
\frac{\partial w}{\partial t}+H\left(g, \frac{\partial w}{\partial g}, t\right)=0 .
$$

* The usual Legendre transform involves only one operator $j(x) \varphi(x)$, composite operators being obtained by multiple functional differentiation at the same point. But this can be extended to include sources for composite operators [5]. 
On the extended phase space Hamilton's equations are supplemented by

$$
\frac{d \phi_{t}}{d \tau}=-\left.\frac{\partial \mathcal{H}_{E}}{\partial t}\right|_{g^{\hat{a}}, \phi}=-\left(\partial_{t} \beta^{\hat{a}}\right) \phi_{\hat{a}}
$$

In other words, with $\tau=t, \frac{d H}{d t}=\frac{\partial H}{\partial t}=\partial_{t} \beta^{\hat{a}} \phi_{\hat{a}}$ and the $t$ dependent Hamiltonian $H(g, \phi, t)$ on $T^{*}(\widehat{\mathcal{M}})$ is not a RG invariant when such subtraction procedures are used. Apart from this difference the analysis is the same as before and the evolution can be described on $T^{*}(\widehat{\mathcal{M}})$ with explicitly $t$ dependent $\beta$-functions.

Finally one might ask, what is the special ingredient of renormalisation group flow which allows it to be written in Hamiltonian form? After all, one cannot expect any vector flow to be expressible as a Hamiltonain flow. The crucial ingredient is the fact that $\frac{d w}{d t}=0$. Thus the renormalisation group is, as the name implies, a symmetry. This leads to the existence of the one-form $\Theta$, which reflects this symmetry, as it is constant along the RG flow, $\mathcal{L}_{\vec{\xi}} \Theta=0$ since $i_{\vec{\xi}} \Theta=0$. In particular, as stated at the beginning of this section, $\frac{d w_{R}}{d t} \neq 0$ and one cannot, in general, get Hamiltonian flow if $\Lambda$ is ignored. Note that

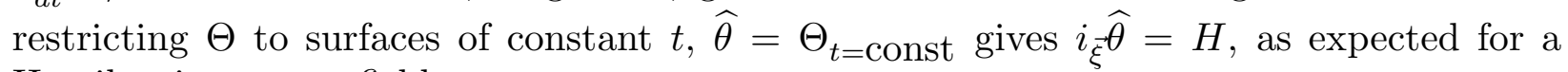
Hamiltonian vector field. 


\section{$\S 4$ An Example - Massive $\lambda \varphi^{4}$}

The construction of the previous section will now be applied to the example of massive $\lambda \varphi^{4}$. We shall use dimensional regularisation and minimal subraction in $D=4-\epsilon$ dimensional Euclidean space. We start with the Lagrangian (10), with a coupling for the identity operator included and $k_{0}$ set to unity,

$$
\mathcal{L}_{0}=\frac{1}{2} \partial_{\mu} \varphi_{0} \partial^{\mu} \varphi_{0}+j_{0} \varphi_{0}+\frac{1}{2} m_{0}^{2} \varphi_{0}^{2}+16 \pi^{2} \frac{\lambda_{0}}{4 !} \varphi^{4}+\Lambda_{0} \mathbf{1}
$$

(the factor $16 \pi^{2}$ is inserted to tidy up some later formulae - it is $\lambda_{0}$ which is really the expansion parameter in $\varphi^{4}$ theory). It will be assumed that $m_{0}^{2}>0$ and that radiative corrections do not change this. A cubic term has been omitted because it can always be eliminated by shifting the field $\varphi_{0} \rightarrow \varphi+$ const. (independently of the scaling that sets $\left.k_{0}=1\right)$.

The scalar field $\varphi_{0}$ has canonical dimension $1-\frac{\epsilon}{2}$ and the couplings $j_{0}, m_{0}^{2}, \lambda_{0}$ and $\Lambda_{0}$ have canonical (mass) dimensions $3-\frac{\epsilon}{2}, 2, \epsilon$ and $4-\epsilon$ respectively. For simplicitly we shall restrict ourselves to the consideration of theories symmetric in $\varphi_{0} \rightarrow-\varphi_{0}$ and set $j_{0}=0$.

The regularisation of the composite operators follows the analysis of [3], but note that here $\Lambda_{0}$ has the opposite sign to that reference. On dimensional grounds one expects

$$
\Lambda_{0}=\kappa^{4-\epsilon}\left(\Lambda+m^{4} F(\lambda, \epsilon)\right),
$$

for some function $F(\lambda, \epsilon)$, analytic in the renormalised coupling $\lambda$ when $\epsilon \neq 0$ (as stated earlier all the renormalised couplings are rescaled to be dimensionless by multiplying by canonical powers of $\kappa$ - thus $m^{2}=\kappa^{-2} \tilde{m}^{2}$ where $\tilde{m}^{2}$ is the usual mass with canonical dimension two).

In dimensional regularisation the renormalised couplings are given in terms of the bare couplings by

$$
\begin{gathered}
m^{2}=\kappa^{-2} z_{2}(\lambda, \epsilon) m_{0}^{2} \quad \lambda=\kappa^{-\epsilon} z_{\lambda}(\lambda, \epsilon) \lambda_{0} \\
\Lambda=\kappa^{-4+\epsilon} \Lambda_{0}-\kappa^{-4} m_{0}^{2} z_{2}^{2}(\lambda, \epsilon) F(\lambda, \epsilon),
\end{gathered}
$$

where $z_{2}$ and $z_{\lambda}$ are the usual renormalisation co-efficients for the mass and $\varphi^{4}$ coupling. Had the linear source been retained, wave function renormalisation would also have entered via $j=\kappa^{-3+\epsilon / 2} z_{1} j_{0}$.

The $\beta$-functions are immediate,

$$
\begin{aligned}
\beta^{\lambda}=\kappa \frac{d \lambda}{d \kappa}=-\epsilon+\beta(\lambda) & \text { with } & \frac{\beta(\lambda)}{\lambda}=z_{\lambda}^{-1} \kappa \frac{d z_{\lambda}}{d \kappa} \\
\beta^{m^{2}}=\kappa \frac{d m^{2}}{d \kappa}=(-2+\delta(\lambda)) m^{2} & \text { with } & \delta(\lambda)=z_{2}^{-1} \kappa \frac{d z_{2}}{d \kappa} \\
\beta^{\Lambda}=\kappa \frac{d \Lambda}{d \kappa}=(-4+\epsilon) \Lambda+m^{4} \zeta(\lambda) & \text { with } & \zeta(\lambda)=(\epsilon-2 \delta(\lambda)) F-\kappa \frac{d F}{d \kappa} .
\end{aligned}
$$


In particular the potential factorises in minimal subtraction, $U\left(\lambda, m^{2}\right)=m^{4} \zeta(\lambda)$. These $\beta$-functions can be supplemented by wave function renormalisation which may be obtained from the $\beta$-function for $j$,

$$
\beta^{j}=\kappa \frac{d j}{d \kappa}=\left(-3+\frac{\epsilon}{2}+\gamma(\lambda)\right) \quad \text { with } \quad \gamma(\lambda)=z_{1}^{-1} \kappa \frac{d z_{1}}{d \kappa}
$$

and $j$ is set to zero after $\gamma(\lambda)$ has been extracted. Of course $\epsilon$ can be put to zero in all of these $\beta$-functions since everything is finite. As is well known, the functions can be developed in a power series in $\lambda$

$$
\begin{aligned}
& \beta(\lambda)=3 \lambda^{2}+o\left(\lambda^{3}\right) \\
& \gamma(\lambda)=\frac{1}{6} \lambda^{2}+o\left(\lambda^{3}\right) \\
& \delta(\lambda)=\lambda-\frac{5}{6} \lambda^{2}+o\left(\lambda^{3}\right) \\
& \zeta(\lambda)=\frac{1}{32 \pi^{2}}\left(1+\frac{\lambda^{2}}{8}+o\left(\lambda^{3}\right)\right)
\end{aligned}
$$

(for the $\lambda^{2}$ term in $\zeta(\lambda)$ see [14].) From equations (66) one has

$$
\left(Z^{-1}\right)_{\hat{a}}^{\hat{b}}=\frac{\partial g_{0}^{\hat{b}}}{\partial g^{\hat{a}}}=\left(\begin{array}{ccc}
-\frac{\epsilon \lambda z_{\lambda}^{-1}}{(-\epsilon \lambda+\beta)} \kappa^{\epsilon} & -\frac{m^{2} \delta z_{2}^{-1}}{(-\epsilon \lambda+\beta)} \kappa^{2} & \frac{m^{4}\{F(\epsilon-2 \delta)+\zeta\}}{(-\epsilon \lambda+\beta)} \kappa^{4-\epsilon} \\
0 & z_{2}^{-1} \kappa^{2} & 2 m^{2} F \kappa^{4-\epsilon} \\
0 & 0 & \kappa^{4-\epsilon}
\end{array}\right) .
$$

Thus the renormalised operators are

$$
\begin{aligned}
& \Phi_{\lambda}=-\left(\frac{\epsilon \lambda z_{\lambda}^{-1} \kappa^{\epsilon}}{-\epsilon \lambda+\beta}\right) \frac{16 \pi^{2}}{4 !} \varphi_{0}^{4}-\left(\frac{m^{2} \delta z_{2}^{-1} \kappa^{2}}{-\epsilon \lambda+\beta}\right) \frac{\varphi_{0}^{2}}{2}+\left(\frac{m^{4}\{F(\epsilon-2 \delta)+\zeta\} \kappa^{4-\epsilon}}{-\epsilon \lambda+\beta}\right) \mathbf{1} \\
& \Phi_{m^{2}}=z_{2}^{-1} \kappa^{2} \frac{\varphi_{0}^{2}}{2}+2 m^{2} F \kappa^{4-\epsilon} \mathbf{1} \\
& \Phi_{\Lambda}=\kappa^{4-\epsilon} \mathbf{1}
\end{aligned}
$$

(strictly speaking the first of these equations should have a term on the right hand side involving the equations of motion for the field [3], but since these operators will only be used inside expectation values, this is omitted).

In order to evaluate expectation values of the renormalised operators one has to interpret the meaning of the operators $\varphi_{0}^{4}$ and $\varphi_{0}^{2}$. In a functional integral approach, these would be the objects appearing in the exponential. When brought down by differentiation they should be considered as time ordered products in expectation values, but of course there are divergences because they consist of products of the field at the same point. These divergences can be treated in dimensional regularisation in the usual way, by using Wick's theorem for free fields and expanding expectation values perturbatively. For example for the operator $\varphi_{0}^{2}(x)$ Wick's theorem gives

$$
\frac{\varphi_{0}^{2}(x)}{2}=\frac{: \varphi_{0}^{2}(x):}{2}+\frac{1}{2} D(0),
$$


where $: \varphi_{0}^{2}(x)$ : denotes normal ordering (with respect to the renormalised mass) and

$$
D(x)=<\varphi_{0}(x) \varphi_{0}(0)>=\int \frac{d^{D} p}{(2 \pi)^{D}} \frac{\mathrm{e}^{-i p \cdot x}}{\left(p^{2}+m^{2}\right)} .
$$

Performing the integral using dimensional continuation yields

$$
D(0)=\frac{1}{4 \pi}\left(\frac{m^{2}}{4 \pi}\right)^{1-\frac{\epsilon}{2}} \Gamma\left(-1+\frac{\epsilon}{2}\right) .
$$

Thus the expectation value is

$$
\frac{1}{2}<\varphi_{0}^{2}>=\frac{1}{8 \pi}\left(\frac{m^{2}}{4 \pi}\right)^{1-\frac{\epsilon}{2}} \Gamma\left(-1+\frac{\epsilon}{2}\right)+o(\lambda)
$$

and the higher order terms can be obtained by expanding the exponential in the functional integral and performing the space integrals (note that $\left\langle: \varphi_{0}^{2}(x):>=o(\lambda)\right.$ is not zero at higher orders because the normal ordering is only defined for free fields). Including the identity term (71) in the expression for $\Phi_{m^{2}}$ one finds that a pole in $F$ cancels the pole in $D(0)$ (as it must do) and, after setting $\epsilon$ to zero

$$
<\Phi_{m^{2}}>=\frac{\kappa^{4} m^{2}}{32 \pi^{2}}\left(-1+\ln m^{2}\right)+o(\lambda),
$$

where the $\overline{M S}$ scheme has been used so as to avoid $-\gamma+\ln 4 \pi$ terms.

Including the next order contribution gives (after some work)

$$
\begin{aligned}
& \phi_{\lambda}=<\Phi_{\lambda}>=\frac{\kappa^{4} m^{4}}{128 \pi^{2}}\left(1-\ln m^{2}\right)^{2}+o(\lambda) \\
& \phi_{m^{2}}=<\Phi_{m^{2}}>=\frac{\kappa^{4} m^{2}}{32 \pi^{2}}\left\{-1+\ln m^{2}+\frac{\lambda}{2}\left(\left(\ln m^{2}\right)^{2}-\ln m^{2}\right)+o\left(\lambda^{2}\right)\right\} \\
& \phi_{\Lambda}=<\Phi_{\Lambda}>=\kappa^{4} \quad \text { to all orders. }
\end{aligned}
$$

These can be derived from the generating functional

$$
w\left(\lambda, m^{2}, \Lambda, t\right)=\frac{m^{4} \kappa^{4}}{64 \pi^{2}}\left(-\frac{3}{2}+\ln m^{2}+\frac{\lambda}{2}\left(1-\ln m^{2}\right)^{2}+o\left(\lambda^{2}\right)\right)+\kappa^{4} \Lambda
$$

by $\phi_{\hat{a}}=\frac{\partial w}{\partial g^{\tilde{a}}}$. The calculation of the order $\lambda^{2}$ terms in $w$ would require extracting the finite part of the three loop diagram (Q).

The Hamiltonian is thus

$$
\begin{aligned}
H(g, \phi) & =\beta^{a}(g) \phi_{a}+\beta^{\Lambda} \phi_{\Lambda} \\
& =\beta(\lambda) \phi_{\lambda}+(-2+\delta(\lambda)) m^{2} \phi_{m^{2}}+\left(m^{4} \zeta(\lambda)-4 \Lambda\right) \phi_{\Lambda} .
\end{aligned}
$$


It is not difficult to prove, from equations (67), (69) and (77), that this is a renormalisation group invariant, $\frac{d H}{d t}=0$, to order $\lambda$. In fact one finds $H=-\frac{\partial w}{\partial t}=-4 w$ when the explicit solutions for the VEV's (77) are substituted into the Hamiltonian. This is a consequence of the fact that all couplings have been scaled to be dimensionless so that the only explicit $\kappa$ dependence in (78) is the trivial volume factor.

The renormalisation group evolution of the vacuum expectation values is thus given by

$$
\begin{gathered}
\frac{d \phi_{m^{2}}}{d t}+\left(\partial_{m^{2}} \beta^{a}\right) \phi_{a}=-\kappa^{D} \partial_{m^{2}} U \\
\frac{d \phi_{\lambda}}{d t}+\left(\partial_{\lambda} \beta^{a}\right) \phi_{a}=-\kappa^{D} \partial_{\lambda} U
\end{gathered}
$$

where the potential is $U(g)=\frac{m^{4}}{32 \pi^{2}}+o\left(\lambda^{2}\right)$. Using the explicit expressions for $\phi_{a}$ in (77) and the $\beta$-functions, (67) and (69), it is a straightforward calculation to check these equations explicitly to order $\lambda$.

Three comments on the analysis presented here are in order.

(i) Using dimensionless couplings will always give $\left.\frac{\partial w}{\partial t}\right|_{g}=4 w$ Thus $H=-4 w$. However, it must be stressed that in applying Hamilton's equations $g^{\hat{a}}$ and $\phi_{\hat{a}}$ in $H(g, \phi)$ must be considered as independent variables. It is only after the theory has been solved and $\phi_{\hat{a}}(g, t)$ determined as a function of $g^{\hat{a}}$ and $t$ that $H$ can be identified with $-4 w$.

(ii) The Hamiltonian, $H=-\langle T\rangle$, is a constant of the motion (RG invariant). This is consisted with the observation that conservation of momentum implies that the energymomentum tensor does not get renormalised (provided the subtraction procedure is compatable with translational invariance [15], as $\overline{M S}$ is). Thus the bare energy-momentum operator is equal to the renormalised one and $\left\langle T_{0}\right\rangle=\langle T\rangle$. (The energy-momentum operator here is, of course, the "improved" operator of reference [15], obtained by coupling the the scalar field $\varphi_{0}$ to the curvature scalar $\mathcal{R}$ of $D$-dimensional space before varying the metric and only setting $\mathcal{R}=0$ afterwards.) How then can the statement that the bare couplings are RG invariants be reconciled with the fact that $-H=<T>=<T_{0}>\neq 0$ ? One answer is that $H$ has canonical mass dimension $D$ despite being a RG invariant. The dimensionless Hamiltonian $\tilde{H}=\kappa^{-D} H$ vanishes at high energy. Perhaps a more rigorous way of stating this is to observe that, as we are using dimensionless renormalised couplings so we could also use dimensionless bare couplings

$$
\tilde{\Lambda}_{0}=\kappa^{4-\epsilon} \Lambda_{0}, \quad \tilde{m}_{0}^{2}=\kappa^{2} m_{0}^{2}, \quad \tilde{\lambda}_{0}=\kappa^{\epsilon} \lambda_{0},
$$

where the quantities with the tildes are the dimensionful ones. One then obtains

$$
\frac{d w\left(g_{0}, \phi_{0}\right)}{d t}=\beta_{0}^{\hat{a}} \phi_{\hat{a}}^{0}+<T_{0}>=0 .
$$

Since $\beta_{0}^{\hat{a}}$ are now non-zero (they are simply the negative of the canonical dimensions of the couplings) this equation is quite consistent. For a regularisation procedure which does not preserve translational invariance one looses the Ward identity that protects the energy-momentum operator from renormalisation and it is no longer necessary to have $<T_{0}>=<T>$. 
(iii) Setting $k_{0}=1$ and then forgetting about it may at first sight seem a little dangerous. After all it is clearly related to wave-function renormalisation and should run like all the other couplings. The fact that the operator $\partial^{\mu} \varphi_{0} \partial_{\mu} \varphi_{0}$ can be ignored with impunity is related to the equations of motion (the Schwinger-Dyson equation). There is always one linear combination of the basic operators $\Phi_{a}^{0}$ which does not get renormalised, namely that corresponding to the "equations of motion" $E_{0}(x)=\varphi_{0}(x) \frac{\delta S_{0}}{\delta \varphi_{0}(x)}$,

$$
E_{0}=-\varphi_{0} \square \varphi_{0}+m_{0}^{2} \varphi_{0}^{2}+\left(16 \pi^{2}\right) \frac{\lambda_{0}}{3 !} \varphi_{0}^{4}
$$

There is a "Ward identity" (the Schwinger-Dyson equation) which ensures that this combination of linear operators does not get renormalised, so $E_{R}=E_{0}$. For this reason one of the operators in the original Lagrangian is always redundant and can be ignored, and here it is $\partial^{\mu} \varphi_{0} \partial_{\mu} \varphi_{0}$ that has been ignored. Strictly speaking though $\partial^{\mu} \varphi_{0} \partial_{\mu} \varphi_{0}$ is not the same operator as $-\varphi_{0} \square \varphi_{0}$ and they should be treated separately. A complete analysis is given in [3], but these complications are omitted here in the interests of clarity. 


\section{$\S 5$ Symmetries}

The notion of a Poisson bracket structure for the renormalisation group evolution, as introduced in section three, immediately raises the question of how symmetries might be implemented on the phase space $\left(g^{\hat{a}}, \phi_{\hat{a}}\right)$. As a first, almost trivial, example of a symmetry consider a $N$-component scalar field $\varphi_{0}^{i}, i=1, \ldots, N$ with Lagrangian

$$
\mathcal{L}_{0}=\frac{1}{2} \sum_{i=1}^{N} \partial_{\mu} \varphi_{0}^{i} \partial^{\mu} \varphi_{0}^{i}+\frac{1}{2} m_{0}^{2} \sum_{i=1}^{N}\left(\varphi_{0}^{i}\right)^{2}+\frac{1}{4 !} \sum_{i j k l} \lambda_{0}^{i j k l} \varphi_{0}^{i} \varphi_{0}^{j} \varphi_{0}^{k} \varphi_{0}^{l} .
$$

There are in general $\frac{1}{4 !} N(N+1)(N+2)(N+3)$ different couplings $\lambda_{0}^{i j k l}$ and each of these could renormalise differently. The different renormalisations of the various fields $\varphi^{i}$ would result in $N$ different renormalised masses $m_{i}^{2}$ as well as the renormalised couplings $\lambda^{i j k l}$ (the renormalised couplings are not all independent parameters, of course, being functions of only $\frac{1}{4 !} N(N+1)(N+2)(N+3)+1$ bare couplings). If however the bare theory enjoys global $S O(N)$ invariance all the $\varphi_{0}^{4}$ couplings reduce to only two, which will be denoted by $\lambda_{0}$ and $\lambda_{0}^{\prime}$. Furthermore, if there are no anomalies, this symmetry survives at the level of the renormalised couplings to give only three renormalised parameters $m^{2}, \lambda$ and $\lambda^{\prime}$. There is a Ward identity which demands that all the renormalised masses $m_{i}^{2}$ must renormalise the same way, i.e. $m_{i}^{2}=z_{m} m_{0}^{2}$, with the same renormalisation constant $z_{m}$ for all the masses $m_{i}^{2}$. Similarly all of the renormalised $\varphi^{4}$ couplings (a priori $\frac{1}{4 !} N(N+1)(N+2)(N+3)$ in number) reduce to only two, $\lambda$ and $\lambda^{\prime}$. Thus the phase space, which is in principle $\frac{2}{4 !} N(N+1)(N+2)(N+3)+2 N$ dimensional, is reduced to being only six dimensional by the symmetry.

A less trivial example is supplied by massless QED coupled to a massless charged scalar field, with Lagrangian

$$
\mathcal{L}_{0}=\frac{1}{4} F_{0}^{2}+i \bar{\psi}_{0} \gamma^{\mu} D_{\mu} \psi_{0}+\left(\tilde{D}_{\mu} \varphi_{0}\right)^{\dagger}\left(\tilde{D}^{\mu} \varphi_{0}\right)+\frac{\lambda_{0}}{4 !} \varphi_{0}^{4},
$$

where the co-variant derivatives are defined by

$$
\begin{aligned}
D_{\mu} \psi_{0} & =\left(\partial_{\mu}+i e_{0} A_{0 \mu}\right) \psi_{0} \\
\tilde{D}_{\mu} \varphi_{0} & =\left(\partial_{\mu}+i \tilde{e}_{0} A_{0 \mu}\right) \varphi_{0},
\end{aligned}
$$

and

$$
F_{0}^{2}=\left(\partial_{\mu} A_{0 \nu}-\partial_{\nu} A_{0 \mu}\right)\left(\partial^{\mu} A_{0}^{\nu}-\partial^{\nu} A_{0}^{\mu}\right) .
$$

A coupling for the identity operator is not necessary since the theory is massless. Also no gauge fixing term is included for the moment because a perturbative analysis will not be used here. In order to avoid volume divergences the theory can be formulated with periodic boundary conditions, i.e. on a four dimensional torus $T^{4}$.

There are three independent couplings, $e_{0}, \tilde{e}_{0}$ and $\lambda_{0}$, but Ward identities force $e_{0}$ and $\tilde{e}_{0}$ to renormalise in the same way so that their renormalised couplings are related to 
the bare counterparts with the same renormalisation constant (i.e. $e=z e_{0}$ and $\tilde{e}=z \tilde{e}_{0}$ where $z$ is the photon wave function renormalisation constant $\left.A_{0}^{\mu}=z A^{\mu}\right)$. Thus

$$
\beta^{e}=\frac{d e}{d t}=\left(z^{-1} \frac{d z}{d t}\right) e \quad \text { and } \quad \beta^{\tilde{e}}=\frac{d \tilde{e}}{d t}=\left(z^{-1} \frac{d z}{d t}\right) \tilde{e}
$$

which implies that $\tilde{e} \beta^{e}=\mathrm{e} \beta^{\tilde{e}}$. The $\beta$-functions are therefore not independent. In particular

$$
\frac{d}{d t}\left(\frac{\tilde{e}}{e}\right)=0
$$

which immediately suggests a change of co-ordinates from $(e, \tilde{e})$ to $r=\sqrt{e^{2}+\tilde{e}^{2}}$ and $\vartheta=\tan ^{-1}(\tilde{e} / e)$, with $0 \leq \vartheta \leq \pi / 2$, so that $\vartheta$ is a RG invariant, $\frac{d \vartheta}{d t}=0$.

The conjugate variables to $e, \tilde{e}$ and $\lambda$ are

$$
\begin{aligned}
\phi_{e} & =\frac{i}{V} \int_{T^{4}} d^{D} x<\bar{\psi} A^{\mu} \gamma_{\mu} \psi> \\
\phi_{\tilde{e}} & =\frac{i}{V} \int_{T^{4}} d^{D} x<A^{\mu} \tilde{D}_{\mu} \varphi>+ \text { Hermitian conjugate } \\
\phi_{\lambda} & =\frac{1}{4 ! V} \int_{T^{4}} d^{D} x<\varphi^{4}>
\end{aligned}
$$

where $V$ is the volume of the torus. The integration over space is kept explicit here so as to mantain gauge invariance which is easily proven by integrating by parts and using the equations of motion. The two VEV's still have canonical mass dimension $D$ due to the volume factors outside the integrals.

Since the theory is massless the potential $U$ vanishes and the RG equations for the VEV's are

$$
\begin{gathered}
\frac{d \phi_{e}}{d t}+\left(\partial_{e} \beta^{e}\right) \phi_{e}+\left(\partial_{e} \beta^{\tilde{e}}\right) \phi_{\tilde{e}}+\left(\partial_{e} \beta^{\lambda}\right) \phi_{\lambda}=0 \\
\frac{d \phi_{\tilde{e}}}{d t}+\left(\partial_{\tilde{e}} \beta^{e}\right) \phi_{e}+\left(\partial_{\tilde{e}} \beta^{\tilde{e}}\right) \phi_{\tilde{e}}+\left(\partial_{\tilde{e}} \beta^{\lambda}\right) \phi_{\lambda}=0 \\
\frac{d \phi_{\lambda}}{d t}+\left(\partial_{\lambda} \beta^{e}\right) \phi_{e}+\left(\partial_{\lambda} \beta^{\tilde{e}}\right) \phi_{\tilde{e}}+\left(\partial_{\lambda} \beta^{\lambda}\right) \phi_{\lambda}=0 .
\end{gathered}
$$

However these are more elegantly expressed in the $(r, \vartheta)$ variables with

$$
\begin{aligned}
& \phi_{r}=\cos \vartheta \phi_{e}+\sin \vartheta \phi_{\tilde{e}} \\
& \phi_{\vartheta}=-r \sin \vartheta \phi_{e}+r \cos \vartheta \phi_{\tilde{e}}
\end{aligned}
$$

and

$$
\begin{aligned}
& \beta^{r}=\cos \vartheta \beta^{e}+\sin \vartheta \beta^{\tilde{e}}=\frac{\beta^{e}}{\cos \vartheta} \\
& \beta^{\vartheta}=0 .
\end{aligned}
$$

Note that since $\cos \vartheta \geq 0$ a positive $\beta^{e}$ always gives a positive $\beta^{r}$, in agreement with the statement that only a non-abelian theory can be asymptotically free. 
The RG evolution of the VEV's in terms of these new variables is now given through Hamilton's equations as

$$
\begin{aligned}
& \frac{d \phi_{r}}{d t}+\left(\partial_{r} \beta^{r}\right) \phi_{r}+\left(\partial_{r} \beta^{\lambda}\right) \phi_{\lambda}=0 \\
& \frac{d \phi_{\vartheta}}{d t}+\left(\partial_{\vartheta} \beta^{r}\right) \phi_{r}+\left(\partial_{\vartheta} \beta^{\lambda}\right) \phi_{\lambda}=0 \\
& \frac{d \phi_{\lambda}}{d t}+\left(\partial_{\lambda} \beta^{r}\right) \phi_{r}+\left(\partial_{\lambda} \beta^{\lambda}\right) \phi_{\lambda}=0 .
\end{aligned}
$$

Since $\beta^{\vartheta}$ vanishes the Hamiltonian

$$
H\left(r, \vartheta, \lambda, \phi_{r} \phi_{\lambda}\right)=\beta^{r}(r, \vartheta, \lambda) \phi_{r}+\beta^{\lambda}(r, \vartheta \lambda) \phi_{\lambda}
$$

is independent of $\phi_{\vartheta}$ and $\vartheta$ is a constant of the motion. In analogy with classical mechanics $\vartheta$ might be called an ignorable co-ordinate, but the roles of co-ordinate and momenta in the RG are really reversed from those of classical mechanics. It is more correct to say the $\phi_{\vartheta}$ is an ignorable expectation value because the Hamiltonian is still a non-trivial function of $\vartheta$. The situation is in fact more involved here than in classical mechanics since the momentum dual to an ignorable co-ordinate in classical mechanics only appears quadratically in the Hamiltonian, whereas the $\vartheta$ dependence of $H$ in equation (96) can be much more complicated. The invariant $\vartheta$ however still plays the same role as an invariant in classical mechanics - since $\{\vartheta, H\}=0$ it generates a symmetry on phase space via the Poisson bracket operation. This can be viewed as the implementation of the Ward identities on phase space.

The fact that one of the expectation values can be eliminated from the phase space can be understood from a physical point of view in the following manner. If the gauge field $A_{0 \mu}$ is rescaled by $A_{0 \mu} \rightarrow \frac{1}{e_{0}} A_{0 \mu}$ then the gauge coupling completely drops out of the matter field terms in the Lagrangian (85) and only appears in the kinetic energy term for the gauge fields, $\frac{1}{4 e_{0}^{2}} F_{0}^{2}$, only the ratio $\vartheta$ appears in the matter field Lagrangian and this is a RG invariant. Defining a new variable $q_{0}=\frac{1}{e_{0}^{2}}$ and forgetting about $\vartheta$ there are now only two expectation values to be considered, $\left.\phi_{q}=\frac{1}{4}<F^{2}\right\rangle$ and $\phi_{\lambda}$. One may expect $\phi_{q} \neq 0$ at large energies, even in a massless theory, since the $\beta$-function for $e$ is positive [16].

There are further interesting aspects of gauge theories when a gauge fixing term is added. If a term $\eta_{0}\left(\partial_{\mu} A_{0}^{\mu}\right)^{2}$ is introduced into the Lagrangian (85) then there is another $\beta$ function, $\beta^{\eta}=\frac{d \eta}{d t}$. In minimal subtraction schemes, the $\beta$-functions for the other couplings are independent of the gauge fixing parameter $\eta$, so $\partial_{\eta} \beta^{r}(r, \vartheta \lambda)=\partial_{\eta} \beta^{\lambda}(r, \vartheta \lambda)=0$. Therefore the expectation value $\phi_{\eta}=\frac{1}{V} \int_{T^{4}} d^{D} x<(\partial . A)^{2}>$ evolves under RG flow according to

$$
\frac{d \phi_{\eta}}{d t}+\left(\partial_{\eta} \beta^{\eta}\right) \phi_{\eta}=0 \quad \Rightarrow \quad \phi_{\eta}(t)=\phi_{\eta}\left(t_{0}\right) \mathrm{e}^{-\int_{t_{0}}^{t}\left(\partial_{\eta} \beta^{\eta}\right) d t}
$$

If $\phi_{\eta}$ is zero at some value of $t$, then it is zero at all values and $\phi_{\eta}$ is another constant constant of the motion. This reflects the fact that $\eta$ plays no physical role in the theory. 
If a renormalisation prescription other than minimal subtraction is used, however, it may not be the case that the other couplings have $\beta$-functions which are independent of the gauge fixing parameter. However, holding to the philosophy that a change in regularisation prescription is just a change in co-ordinates, there must be some quantity that is a RG invariant, i.e. the co-ordinate transform of the dimensional regularisation co-ordinate $\eta$ it may look messy in the new co-ordinates but it must exist.

In conclusion it would seem that, just as in classical mechanics, the Hamiltonian framework is a very powerful one for the discussion of symmetries, which play such a central role in all discussions of quantum field theory. 


\section{$\S 6$ Conclusions}

Before summarising the main results of this paper a few comments will be made about the global topology of the space of couplings $\widehat{\mathcal{M}}$, as promised in the introduction. Consider for definiteness massless QED with the only coupling being the electron charge $e$ (the identity operator can be ignored). It is really $\alpha=\frac{e^{2}}{\hbar c}$ (or $1 / \alpha$ ) which is the important parameter, and $\alpha$ must be positive since a negative value would mean that the theory would be unstable, as pointed out by Dyson [17]. Thus $\alpha=0$ is not an analytic point and one cannot continue, even infinitesimally, to $\alpha<0$. Similarly $1 / \alpha=0$ cannot be an analytic point. The manifold $\mathcal{M}$, the positive real line, has two boundary points both of which are non-analytic points of the theory. For the higher dimensional case points of non-analyticity are clearly also of central importance. Such points might be isolated or might form sub-manifolds of $\widehat{\mathcal{M}}$ with dimension $k<n$. If the non-analytic points were to form a sub-manifold of co-dimension one (a hypersurface $k=n-1$ ), then this would act as an effective boundary $\partial \widehat{\mathcal{M}}$. In any event, it is clear that any understanding of the global topology of $\widehat{\mathcal{M}}$ will be inextricably linked with an understanding of the points of non-analyticity.

In summary it has been argued that the renormalisation group evolution of couplings and vacuum expectation values can be described as a Hamiltonian flow on the $2 n$ dimensional phase space $T^{*}(\widehat{\mathcal{M}})$ with the Hamiltonian given by

$$
H(g, \phi, t)=\beta^{a}(g, t) \phi_{a}+(U(g, t)-D \Lambda) \phi_{\Lambda},
$$

which can be identified with minus the vacuum expectation value of the trace of the energy-momentum tensor.

The natural variables canonically conjugate to the couplings are the expectation values, $\phi_{a}=\partial_{a} w(g, t)$, where $w(g, t)$ is the generating functional or free energy density. For theories with massive couplings the cosmological constant plays a central role, since its $\beta$-function $\beta_{\Lambda}=U(g, t)-D \Lambda$ gives rise to a potential which acts as an effective force in the RG evolution of the VEV's

$$
\frac{d \phi}{d t}=-\kappa^{D} d U(g, t)
$$

where $\phi=\phi_{a} d g^{a}$ and $d U=\partial_{a} U d g^{a}$.

The RG evolution of any function $A(g, \phi, t)$ can be determined from the Hamiltonian

$$
\frac{d A}{d t}=\{A, H\}+\left.\frac{\partial A}{\partial t}\right|_{g, \phi}
$$

In particular the RG evolution of the Hamiltonian itself is given by

$$
\frac{d H}{d t}=\frac{\partial H}{\partial t}=\partial_{t} \beta^{a}(g, t) \phi_{a}+\partial_{t} U(g, t) \phi_{\Lambda},
$$


and $H$ is a $\mathrm{RG}$ invariant if a subtraction procedure is chosen so that the $\beta$-functions are independent of $\kappa$. Alternatively the cosmological constant can be omitted and the RG evolution of the Hamiltonian $h$ on $T^{*}(\mathcal{M})$ is given by

$$
\frac{d h}{d t}=\frac{\partial h}{\partial t}=\partial_{t} \beta^{a}(g, t) \phi_{a}+\kappa^{D}\left(D U(g, t)+\partial_{t} U(g, t)\right)
$$

which can only be a RG invariant if both $U=0$ and the $\beta$-functions have no explicit $\kappa$ dependence.

The RG equation for the $N$-point Green functions is

$$
\begin{aligned}
\frac{\partial}{\partial t} G_{a_{1} \cdots a_{N}}^{(N)}(g, \phi, t) & +\beta^{b} \partial_{b} G_{a_{1} \cdots a_{N}}^{(N)}(g, \phi, t)+\sum_{i=1}^{N}\left(\partial_{a_{i}} \beta^{b}\right) G_{a_{1} \cdots a_{i-1} b a_{i+1} \cdots a_{N}}^{(N)}(g, \phi, t) \\
& =\left(\phi_{c}\left(\partial_{b} \beta^{c}\right)+\kappa^{D} \partial_{b} U\right) \frac{\partial}{\partial \phi_{b}} G_{a_{1} \cdots a_{N}}^{(N)}(g, \phi, t) .
\end{aligned}
$$

The crucial ingredient that gives rise to Hamiltonian flow is the underlying symmetry of the renormalisation group, reflected in the fact that $\frac{d w}{d t}=0$. Ward identities give rise to further constants of the motion which generate symmetries on phase space via the Poisson bracket structure.

The RG equation for the generating functional $w(g(t), t)$ can be interpreted as a Hamilton-Jacobi equation

$$
\left.\frac{\partial w}{\partial t}\right|_{g}+H\left(g, \frac{\partial w}{\partial g}, t\right)=0
$$

Table 1 provides a summary of the correspondence between concepts in quantum field theory or statistical mechanics and classical mechanics

It is a pleasure to thank William Deans, Chris Ford, Bill McGlinn, Charles Nash, and Lochlainn O'Raifeartaigh for many useful conversations about the RG. It was Denjoe O'Connor and Chris Stephens who first suggested to me that a symplectic structure should play an important role in quantum field theory and statistical mechanics, with the VEV's being conjugate momenta, and that the RG equation should be viewed as a HamiltonJacobi like equation. This work would not have been possible without their prompting. I also wish to thank the Alexander von Humboldt foundation whose financial support enabled me to carry out most of the calculations of section four. 


\section{References}

[1] E. Brézin, J.C. Le Guillou and J. Zinn-Justin, Phys. Lett. 44A (1973) 227;

Phys. Rev. B 10 (1973) 892

[2] Geometry, The Renormalisation Group And Gravity D. O'Connor and C.R. Stephens, in Directions In General Relativity,

Ed. B.L. Hu, M.P. Ryan Jr., and C.V. Vishveshwava

Proceedings of the 1993 International Symposium, Maryland, Vol 1, C.U.P. (1993)

[3] L.S. Brown, Ann. Phys. 126 (1979) 135

L.S. Brown and J.C. Collins, Ann. Phys. 130 (1980) 215

[4] D.J. Wallace and R.K.P. Zia, Phys. Lett. A48 (1974) 565

D.J. Wallace and R.K.P. Zia, Ann. Phys. 92 (1975) 142

[5] A.N. Vasil'ev, A.K. Kazanskii and Yu. M. Pis'mak, Teor. i Math. Fiz. 19 (1974) 186

J.M. Cornwall, R. Jackiw and E. Tomboulis, Phys. Rev D 10 (1974) 2428

G.M. Shore, Nucl. Phys. B362, (1991), 85

[6] A.B. Zamolodchikov, Rev. Math. Phys. 1, (1990), 197

[7] Ta-Pei Cheng and Ling-Fong Li, Gauge Theory Of Elementary Particle Physics (1984) O.U.P.

[8] B.P. Dolan, Int. J. of Mod. Phys. A 9 (1994) 1261

[9] V.I. Arnold, Mathematical Methods Of Classical Mechanics 2nd. Ed. Graduate Texts in Mathematics 60, (1989) Springer

[10] M. Lässig, Nucl. Phys. B334, (1990), 652

[11] D. O'Connor and C.R. Stephens, unpublished

[12] I. Jack and H. Osborn, Nucl. Phys. B343, (1990), 647

[13] G. Jona-Lasinio, Nuovo Cimento 34 (1964) 1790

[14] S.J. Hathrell, Ann. Phys. 139 (1982) 136

[15] C.G. Callan Jr., S. Coleman and R. Jackiw, Ann. Phys. 59 (1970) 42

[16] V.A. Miransky, Il Nouvo Cimento 90 (1985) 149

[17] F.J. Dyson, Phys. Rev. 85 (1952) 631 
A Quantum Field Theory-Classical Mechanics Dictionary

\begin{tabular}{|l|l|}
\hline $\begin{array}{l}\text { Quantum Field Theory or } \\
\text { Statistical Mechanics }\end{array}$ & Classical \\
\hline $\begin{array}{l}\text { Couplings } g^{a}(t) \\
\beta \text {-functions } \beta^{a}(t)\end{array}$ & $\begin{array}{l}\text { Co-ordinates } q^{a}(t) \\
\text { Velocities } \dot{q}^{a}(t)\end{array}$ \\
Vacuum expectation values $\phi_{a}(t)$ & $\begin{array}{l}\text { Momenta } p_{a}(t) \\
\text { Bare couplings } \quad\left(g_{0}^{a}, \phi_{a}^{0}\right)\end{array}$ \\
Initial point $\quad\left(q_{0}^{a}, p_{a}^{0}\right)$
\end{tabular}

